\title{
AN ENHANCED ROUTE FAILURE RECOVERY MODEL FOR MOBILE AD HOC NETWORKS
}

\author{
${ }^{1}$ P.R. Jasmine Jeni, ${ }^{2}$ A. Vimala Julie and ${ }^{3}$ A. Messiah Bose \\ ${ }^{1}$ SRM University, Chennai, Tamilnadu, India \\ ${ }^{2}$ SRM University, Chennai, Tamilnadu, India \\ ${ }^{3}$ Chemtrols Industries Pvt Limited, Chennai, Tamilnadu, India
}

Received 2014-01-10; Revised 2014-02-11; Accepted 2014-04-08

\begin{abstract}
In Mobile Ad Hoc Networks (MANET), change in topology of the network occurs due to the mobility factor of the nodes leading to the extension in size of the network. The extension of network size happens due to the entry of nodes into the network. As the topology changes, link failure between the nodes takes place due to several reasons like channel interference and dynamic obstacles etc that give rise to severe performance degradation. In traditional AODV, the link failure is overcome by re-routing from the source node which is a time consuming process that increases the overhead of the nodes. Also in case of multiple link failures, there are chances for loss of data packet. Maintaining the performance of the network dynamically during link failure, specifically in case of long data transfer such as the stream of voice data, is a challenging problem. In order to overcome such performance related issues, we developed the Local Link Failure Recovery algorithm (LLFR) for Ad hoc networks that establishes recovery from link failures spontaneously at the point of link breakage. In such cases, a reliable link failure recovery is the main criteria that will determine the performance of the network in terms of Quality of Service (QoS). The LLFR is deployed in each node collects RREP in the RREP Buffer Table (RBT) stack in the highest order of signal strength, which gets triggered during link failures. Once a link failure is detected, the intermediate node searches for an alternate path around the faulty area by choosing the first RREP that is stacked in the RBT and establishes a new route to the intended destination for sending the data packets without any time delay. The simulation results show that the performance parameters like packet delivery ratio, throughput, average end to end delay and routing overhead are better compared to the traditional AODV and other link failure recovery techniques.
\end{abstract}

Keywords: MANET, Routing, Link Failure, Signal Strength

\section{INTRODUCTION}

In wireless communication systems, the mobile nodes or users are deployed independently and are free to move. Due to this reason, the network topology changes rapidly and unpredictably over time, thereby changing its links to neighboring nodes frequently. As the network is decentralized, establishment of communication is extremely challenging due to the dynamic topology. Since the routing process is associated within the mobile nodes, the routine exercises pertaining to the network such as exploring the network topology and transmitting the data are performed by the node itself.
A MANET (Corson and Macker, 1999) is a collection of self determining nodes that are mobile and communicate via confined wireless bonds. The routing protocols in MANET are categorized into three types, namely pro-active, reactive and hybrid routing protocols. In proactive routing protocols, every node in the network maintains the routing table that is updated regularly. The nodes exchange the topology information to keep the routing table with latest notifications leading to high overhead, as they are flooded with information pertaining to unknown links.

Ad hoc On-demand Distance Vector, (AODV) (Perkins and Royer, 1999; Perkins et al., 2003) is a

Corresponding Author: P.R. Jasmine Jeni, SRM University, Chennai, Tamilnadu, India 
reactive routing protocol used in wireless networks that discovers a route to destination on demand. AODV requires each node to maintain a routing table containing the discovered path information. AODV is capable of creating fresh routes whenever a route error occurs. The advantages of AODV is that, it uses sequence numbers to determine the freshness of the route thereby preventing loop formation and doesn't create overhead unnecessarily during communication.

MANET's have become highly adaptable to all the groups, as human society relies on portability of devices which enhances the importance of wireless connectivity in work places, offices, colleges, hotels etc. Routing in MANET (Taneja and Kush, 2010) is always a distinctive task and it becomes a challenge to have an appropriate routing scheme when the network size grows more sizeable. Owing to the mobility of nodes in a wireless network, the network topology changes and the route length between the source to destination increases. When the link between the nodes in a network suffers due to failures, the reactive protocols like DSR and AODV generally drops the original route and triggers a new route discovery process causing overhead in local route discovery. The re-routing is an energy consuming process that heaps the overheads on the nodes. The motivation of this study is to overcome link breakages, by recovering link failures locally and spontaneously thereby establishing routes without losing the data packets.

In this study, we introduce an enhanced novel Local Link Failure Recovery algorithm (LLFR) for recovering from link failures locally in Ad hoc networks. When a link failure occurs due to faint signal between nodes, the route has to be configured and repaired spontaneously so that there is no data loss and the data stream is fully transferred. When a link failure is detected by a node, the Local Link Failure Recovery (LLFR) mechanism deployed in each node arrives on an alternate path from that intermediate node which did not receive the RREP i.e. the failed node. The LLFR then updates the alternate path to source and sends the data packets to the destination much faster, instead of dropping the whole route and discovering a new route to the destination. The over head among nodes are significantly reduced as the failure recovery is done locally. The packet delivery ratio also increases, as preventive measures for safe landing of data packets to the destination are taken in the new route, by keeping a constant tab on the signal strength of neighboring nodes. Using stimulation we found that this mechanism exhibits better efficiency by overcoming the overhead issues during link failures.

This research paper presents the related work in section 2 , the proposed system description in section 3 , the results in section 4 and the conclusion in section 5 .

\section{RELATED WORKS}

AODV is widely used by mobile nodes in ad hoc network for routing purposes. It provides hop by hop routing using route discovery and route maintenance schemes (Cigdem and Kravets, 2006). It also provides local repair to recover the route when a node detects the broken link in an active route by rerouting entirely and this process consumes comparatively more time.

In AODV, a route discovery phase is implemented on-demand when a route fails and the route maintenance phase starts by flooding a route error message over the network. By its architecture, the AODV increases its route discovery process quite frequently thereby increasing the overhead. To improve the problem of overhead caused during route discovery process, several studies has been established like the partial reestablishment approach and the multipath approach. In partial re-establishment approach, the routing protocol finds an alternate route during the route maintenance phase. In multipath approach, the routing protocol establishes many routes during the route discovery phase. As the Multipath AODV (Marina and Das, 2006; Tsirigos and Haas, 2001) establishes possible number of multiple routes regardless the route efficiency, there can be a large number of inefficient routes associated with the route discovery process which leads to enormous routing overhead. The packet drop and latency is more in multipath AODV, as this protocol depends on unused routes too. Even though multipath routing is significantly better than single path routing, the performance advantage is too small.

The Bypass-AODV (Cigdem and Kravets, 2006) uses cross-layer MAC-notification to determine mobilityrelated link failure and sets up a bypass between the broken link end- nodes via an alternative node while keeping the remaining nodes of the route as it is. The performance of Bypass-AODV is enhanced compared to the traditional AODV, as the error recovery phase is eliminated thereby reducing routing overheads and packet drop ratio. The Bypass-AODV transmits the packets via the newly constructed bypass route eluding packet drop. The performance of Bypass-AODV is best at high node density, when the distance between the endnodes is greater than or equal to three hops. At low density of nodes where node connectivity is low, Bypass-AODV is not suitable due to occurrence of collision.

Mobility prediction and routing ( $\mathrm{Su}$ et al., 2000) is used to overcome route failures by obtaining local route repair, when a link break is about to occur. The mobility information from each node is used to predict 
the instant when the link between two neighbors will break. The location and motion pattern of each neighboring node is recorded via an extended-hello message that is generated from nodes belonging to the active routes. The information pertaining to location and mobility of the nodes is constantly reproduced between neighbors and hence incurs huge overheads. A new QoS routing protocol (Ramadoss et al., 2014) is proposed which provides spanning tree based path selection by avoiding congestion, balancing the load and energy paving way to avoid data loss simultaneously minimizing the communication overhead without reducing the network performance.

A good performance comparison of DSR and AODV can be found in (Das et al., 2000). The work in (Babbitt et al., 2009) is a good example of self route selecting scheme for the sake of reliability. When a data packet is sent from a source to a destination, each node competes for self selection based on back-off delay in this scheme. Although there are several mechanisms to overcome link breakage and link failure recovery, each has its own limitations. We propose that localization of link failure recovery will reduce the overhead of route discovery and is essential for adhoc routing protocols to improve its QoS parameters.

\section{DESIGN OF LLFR}

It has been widely accepted that routing in MANET is a challenge, as the network size increases. The highly dynamic and unstable nature of mobile nodes in large scale Ad Hoc networks causes radio links to break frequently. Wireless networks are highly liable to suffer from route breaks due to several reasons such as signal interference, data collision, faint environment, node mobility etc., The Local Link Failure Recovery Algorithm (LLFR) deployed in each nodes present in the network (i) performs local route recovery minimizing data packet loss during link failures in ad hoc network (ii) overcomes issues pertaining to overhead caused to nodes during link failures (iii) Improves QoS parameters like the packet delivery ratio, average end to end delay and throughput compared to its predecessors. The schematic representation of LLFR is given in Fig. 1.

The session inducted by the LLFR consists of spontaneous initiation of the LLFR and checking the RBT for alternate path without disturbing the network setup. The neighboring node with highest signal strength is chosen to forward the data. When a link failure is detected by a node, it immediately triggers the LLFR to explore an alternate route to the destination simultaneously having a vigil on the signal strength of successive links. The LLFR algorithm comprises a
RREP Buffer Table which stores the RREP's received from the neighboring downstream nodes in ascending order of signal strength. The received signal strength is the MAC layer information used by the routing layer of the nodes through cross layer interaction. The RSSI is the received signal strength indication, which is used to determine the amount of radio energy in the channel. RSSI is possible to estimate the relative stability of the link based on recent and current received signal strength. The overhead on each node is drastically reduced due to non transmission of RERR packet to source node. The schematic of an instance with 7 nodes in a network including a source and a destination is shown below in Fig. 2 .

Let us consider that node $\mathrm{j}$ receives a RREQ packet from its upstream node $\mathrm{US}_{\mathrm{n}} \mathrm{i}$. The node $\mathrm{j}$ immediately checks the destination ID of the received RREQ packet and responds to the node $\mathrm{US}_{\mathrm{n}} \mathrm{i}$ with a RREP packet only if the ID of node $\mathrm{j}$ matches with the destination ID. If the ID of node $\mathrm{j}$ doesn't match with the ID of destination ID, then node $\mathrm{j}$ forwards the RREQ packet to its neighboring nodes. Once the node $\mathrm{j}$ receives the RREP packet from its neighboring node e.g.,: Downstream node $\mathrm{k}, \mathrm{DS}_{\mathrm{n}} \mathrm{k}$ and downstream node $1, \mathrm{DS}_{\mathrm{n}} 1$, it stores the information about the received RREP packet in the RREP Buffer Table (RBT) and the node ' $\mathrm{j}$ ' then sends the RREP packet to node ' $U S_{\mathrm{n}} \mathrm{i}$ '. The node ' $U S_{\mathrm{n}} \mathrm{i}$ ' forwards the data packet to the node $\mathrm{j}$ upon receiving the RREP packet from node ' $\mathrm{j}$ '. The selected path for data transmission in this case is $\mathrm{US}_{\mathrm{n}} \mathrm{i} \rightarrow \mathrm{j} \rightarrow \mathrm{DS}_{\mathrm{n}} \mathrm{k}$. Suppose if a link failure occurs between the node ' $\mathrm{j}$ ' and node ' $\mathrm{DS}_{\mathrm{n}} \mathrm{k}$ ', the node 'j' will check various RREP's received in the RBT for an alternate route to reach the destination. As the alternate route selection is depending on the signal strength of the neighboring node, the links with low signal strength will be discarded and safe landing of data packet is ensured.

For example, in RREP Buffer Table if the signal strength is high for the node ' $\mathrm{DS}_{\mathrm{n}} \mathrm{l}$ ', the new route to reach the destination node to deliver the data packet is $\mathrm{j} \rightarrow \mathrm{DS}_{\mathrm{n}} \mathrm{l} \rightarrow-$---- $\rightarrow$ Destination node. Simultaneously the node ' $\mathrm{j}$ ' will send the route update message to the source node through the upstream node. When the selected $\mathrm{DS}_{\mathrm{n}}$ is not a destination node, link failure recovery process will continue. In case of the existing routing protocols, once the link failure occurs, the intermediate node will send the route error message to the source node and again initiates the route discovery process for the same data packet reducing the performance of the network gradually. For instance, when the node i receives the RREQ from its $\mathrm{DSj}$, it measures the signal strength of the RREQ packet and in the reverse path it updates the signal strength parameter of its RREP to its DSj. 


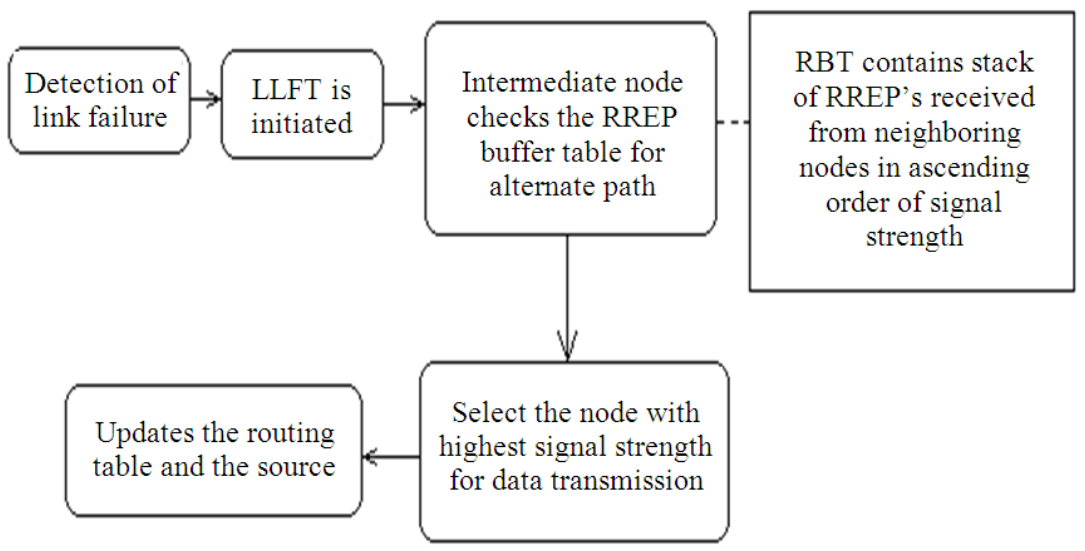

Fig. 1. LLFR model

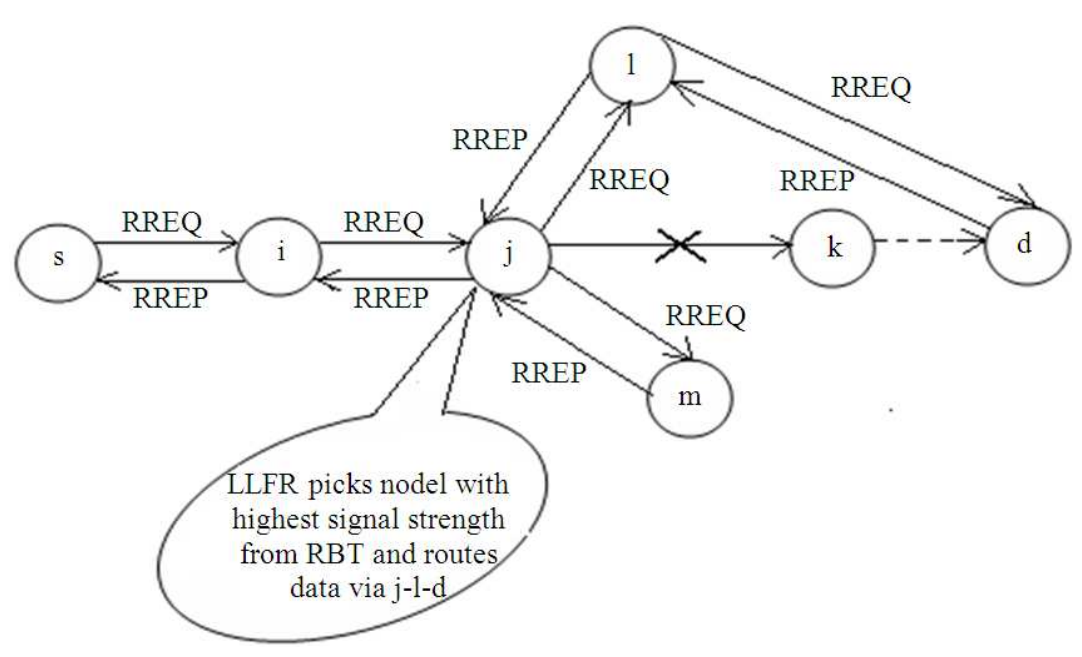

Fig. 2. Schematic of a network for an instance

\section{IMPLEMENTATION OF LLFR}

The LLFR algorithm implemented with AODV routing protocol is described below:

1: If link failure detected then

2: Go to step 4

3: Else data packet is transmitted

4: LLFR is activated

5: The intermediate node receives RERR act as the source node

6: Select the first entry in the RBT stack as the immediate node

7: Create alternate path using RBT information in each node

8: Transmit data packets via alternate path to destination 9: Update the new route to the source node
The LLFR deployed in every node updates the RBT with RREP packet in ascending sequence of highest signal strength from relevant downstream nodes. So when a link failure is detected, the foremost RREP stored in the RBT will be chosen as the next downstream node and this process continues until reaching the destination. The alternate path is updated with the source node and the routing table of all relevant nodes. The Local Link Failure Recovery Algorithm with AODV routing protocol is implemented and evaluated using the Network Simulator (NS 2, version 2.32). The NS2 provides substantial support for simulation of wireless networks and is more user friendly meeting diverse needs. NS2 is a cost effective solution that is alternate to real world network used to evaluate and analyze the behavior of various network design. The parameters used in our simulation are shown in Table $\mathbf{1 .}$ 
Table 1. Simulation parameters

\begin{tabular}{ll}
\hline Radio propagation model & Two Ray ground \\
\hline Mobility Model & Random Way Point \\
MAC Type & MAC802.11n \\
Antenna model & Omni Antenna \\
Number of mobile nodes & 100 \\
Routing protocol & AODV \\
Terrain & $1500 \mathrm{~m} \times 500 \mathrm{~m}$ \\
Length of data packets & 512 bytes \\
Simulation time & 500 milliseconds \\
Local repair wait time & 0.15 milliseconds \\
maximum RREQ time out & 10 milliseconds \\
RREP wait time & 1 millisecond \\
\hline
\end{tabular}

\section{RESULTS}

The simulation results of Local Link Failure Recovery Algorithm (LLFR) incorporated in AODV routing protocol is given below.

\subsubsection{Packet Delivery Ratio}

PDR is the ratio between the numbers of packets received by the application layer of destination node to the number of packets sent by the application layer of source node.

$$
\mathrm{PDR}=\frac{\mathrm{P}_{\mathrm{recd}}}{\mathrm{P}_{\text {sent }}} \times 100
$$

where, PDR is packet delivery ratio, $\mathrm{P}_{\text {recd }}$ represent the total number of data packets received and $P_{\text {sent }}$ represent the total number of data packets sent.

\subsubsection{Throughput}

Throughput is the number of bits transmitted per unit second over a communication channel. Below is results of LLFR compared to the traditional AODV routing protocol.

\subsubsection{Average End-to-End Delay}

End-to-end delay is defined as the time taken for a data packet to be transmitted across a wireless network from the source to destination.

The below result show the average end to end delay of the LLFR with AODV routing protocol.

\subsubsection{Protocol Overhead}

Protocol overhead refers to the number of routing messages requested when a data packet is successfully delivered to the destination.

\section{PERFORMANCE EVALUATION}

The performance of the LLFR with AODV is compared with traditional AODV routing protocol for its packet delivery ratio, throughput, overhead and end to end delay. The simulation results of packet delivery ratio of AODV with LLFR routing protocol as referred in Fig. 3 has increased when compared to traditional AODV routing protocol during link failures. It is also observed that the PDR with LLFR is relatively consistent or even better during link failures, as compared to AODV in such situations. When there are more failure nodes, the routing protocol with LLFR tends to have a better PDR compared to the AODV. The average delay of transmitted data packet is calculated by dividing the total delay by the number of packets arrived at the destination. The simulation results in Fig. 4 show that the throughput of AODV with LLFR is significantly better compared to AODV in the event of link failure. The LLFR achieves better throughput when compared to the other case, as the alternate path chosen by the LLFR is reliable leading to better throughput. There is negligible chance of data packet loss in case of stream of data such as voice or video as the intermediate node in no time triggers the LLFR algorithm and starts routing the data via a reliable alternate path.

The average end to end delay is reduced considerably in the LLFR as referred in Fig. 5 when compared to traditional AODV routing protocol in conditions of node failure. This has been achieved by allowing the intermediate node to spontaneously choose the alternate route during the link failure. Here, the data transmission time after failure is reduced, as the RBT readily has the RREP with highest signal strength of the next forwarder ready in the stack. The LLFR has reasonably lesser overhead when compared to AODV as referred in Fig. 6. In traditional AODV, mobile nodes respond to link failures with numerous messages that are flooded across the network to maintain an active route in AODV, resulting in high overheads. The routing Protocol with LLFR has the best overhead performance because of its uniqueness in spontaneously responding to link failures. Even though the overhead of LLFR is reasonably significant, the overhead of the LLFR incorporated Ad hoc network with multiple link failures is far better. 
P.R. Jasmine Jeni et al. / Journal of Computer Science 10 (8): 1561-1568, 2014

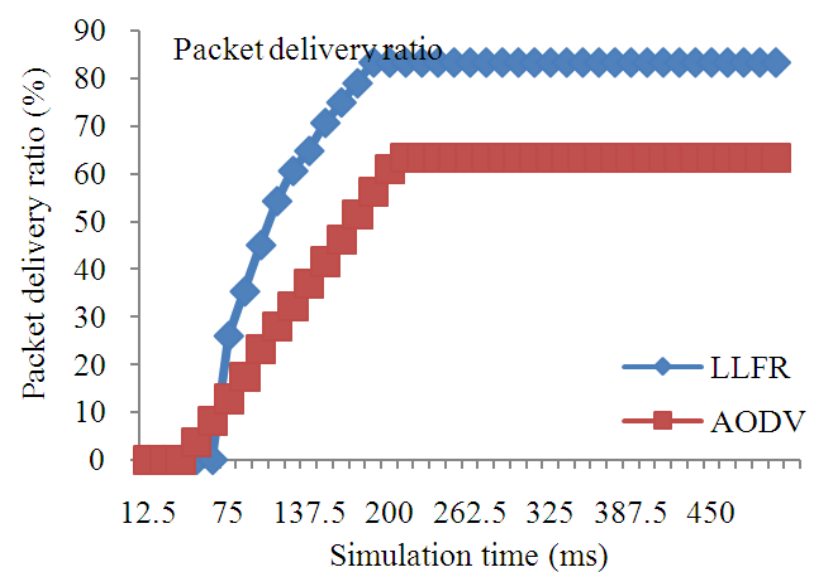

Fig. 3. Packet delivery ratio

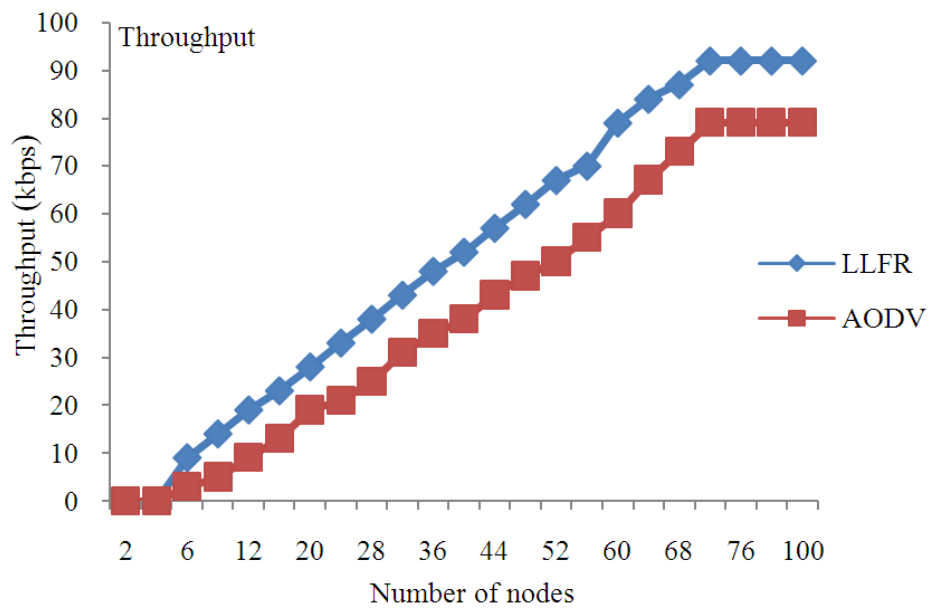

Fig. 4. Throughput

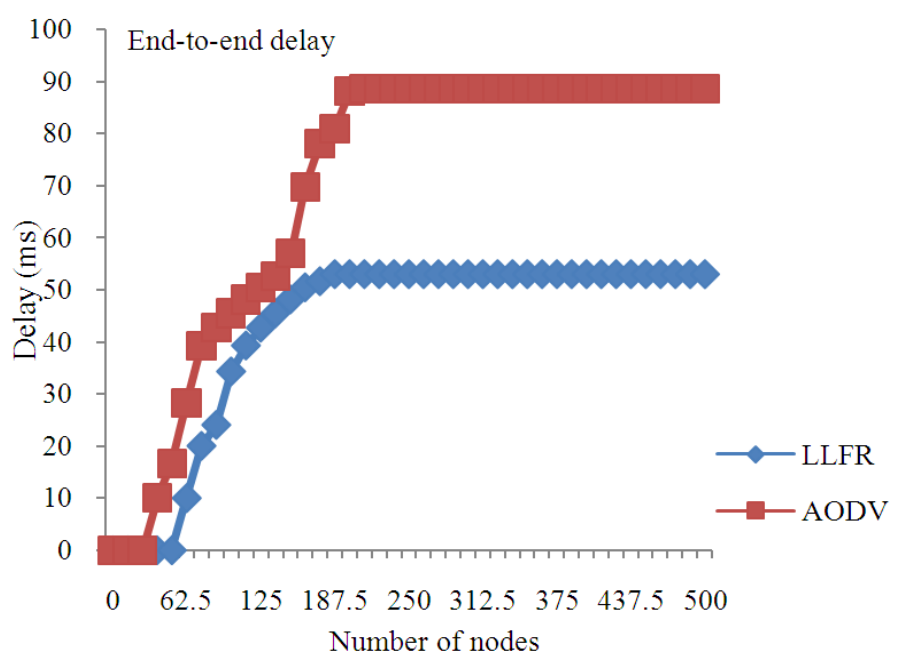

Fig. 5. End to end delay 


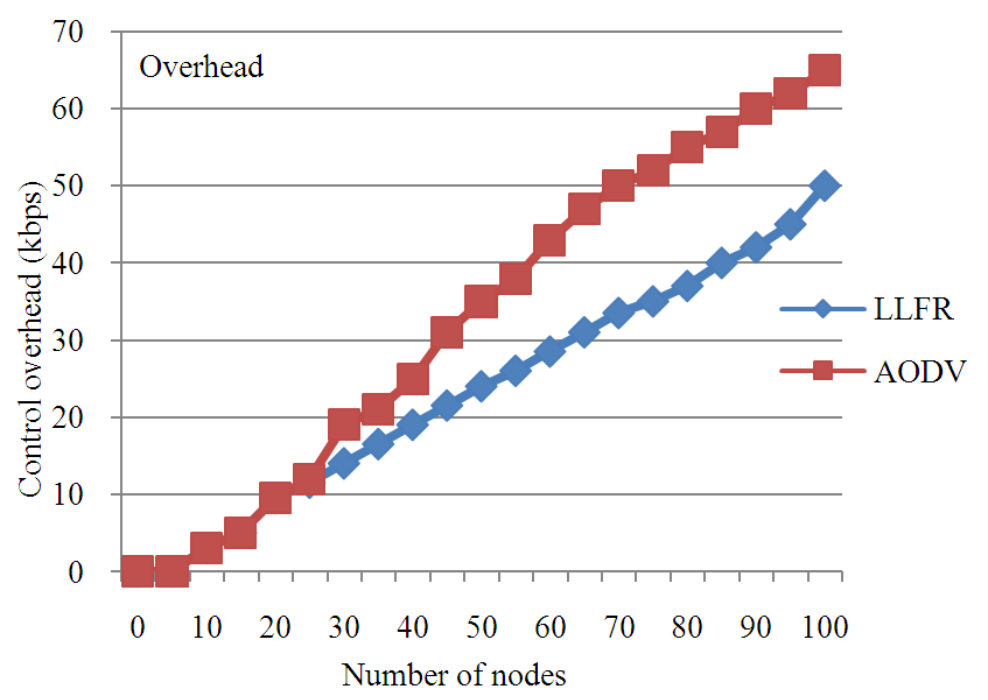

Fig. 6. Routing overhead

\section{CONCLUSION}

A novel scheme for Ad hoc networks to recover from link failure called the Local Link Failure Recovery algorithm (LLFR) with AODV routing protocol is implemented in this study. The simulation results obtained indicate the improved efficiency of the LLFR with AODV routing protocol by showing significant improvement in the QoS demands of today's wireless Ad Hoc network with single or multiple link failures. The performances of routing protocols also rely up on the number of nodes or participants in the network. Here the performance of LLFR algorithm incorporated with AODV routing protocol is compared with traditional AODV in terms of packet delivery ratio, routing overhead, throughput and average end to end delay and found significantly better in all aspects. This is achieved because the LLFR is activated spontaneously during link failure thereby reducing the possibility of data packet loss. The overhead of AODV with LLFR is significantly low compared to traditional AODV, as the functionality is need-based, overcoming the unnecessary overheads caused by the routing nodes. The overhead is even less in case of more than one link failures as the traditional AODV takes more time to recover from multiple link failures.. The end to end delay is improved using LLFR as the spontaneous recovery of route takes place on the link failure occurrence. LLFR once activated avoids further delay in transmitting the data packets as the link stability in terms of signal strength is taken care by the LLFR itself for further transmission. i.e., The LLFR takes care of the data packets for the safe transit to the destination without further delay. The simulation results show that the AODV routing protocol incorporated with LLFR effectively increases the throughput and reduces delay when compared to traditional AODV routing protocol. An analysis of the energy in these networks and lifetime increment issue can be taken into account as part of the future work.

\section{REFERENCES}

Babbitt, T., C. Morrell and B. Szymanski, 2009. Selfselecting reliable path routing in diverse wireless sensor network environments. Proceedings of IEEE International Symposium on Computers and Communication, Jul. 5-8, IEEE Xplore Press, Sousse, pp: 1-7. DOI: 10.1109/ISCC.2009.5202268

Cigdem, S. and R. Kravets, 2006. Bypass routing: An on-demand local recovery protocol for ad hoc networks. Ad Hoc Netw., 4: 380-397. DOI: 10.1016/j.adhoc.2004.10.004

Corson, S. and J. Macker, 1999. Mobile Ad Hoc Networking (MANET) Routing protocol performance issues and evaluation. University of Maryland.

Das, S.R., C.E. Perkins and E.M. Royer, 2000. Performance comparison of two on-demand routing protocols for ad hoc networks. Proceedings of the 9th Annual Joint Conference of the IEEE Computer and Communications Societies, (CCS '00), IEEE Xplore Press, Tel Aviv, pp: 3-12. DOI: 10.1109/INFCOM.2000.832168 
Marina, M.K. and S.R. Das, 2006. Ad hoc on-demand multipath distance vector routing. Wireless Commun. Mobile Comput., 6: 969-988. DOI: 10.1002/wcm.432

Perkins, C. and E.M. Royer, 1999. Ad-hoc on-demand distance vector routing. Proceedings of the 2nd IEEE Workshop on Mobile Computing Systems and Applications, Feb. 25-26, IEEE Xplore Press, New Orleans, LA., pp. 90-100. DOI: 10.1109/MCSA.1999.749281

Perkins, C., E.B. Royer and Chakeres, 2003. Ad Hoc On Demand Distance Vector (AODV) routing. IETF Internet draft. University of California.
Ramadoss, P., S.M. Yakub and S. Annaji, 2014. A preemptive link state spanning tree source routing protocol for mobile ad hoc networks. J. Comput. Sci., 10: 85-90. DOI: 10.3844/jcssp.2014.85.90

Su, W., S.J. Lee and M. Gerla, 2000. Mobility prediction and routing in ad hoc wireless networks. Int. J. Netw. Manage., 11: 3-30. DOI: 10.1002/nem.386

Taneja, S. and A. Kush, 2010. A survey of routing protocols in mobile ad hoc networks. Int. J. Innovat. Manage. Technol., 1: 279-285.

Tsirigos, A. and Z.J. Haas, 2001. Multipath routing in the presence of frequent topological changes. IEEE Commun. Magazine, 39: 132-138. DOI: $10.1109 / 35.965371$ 